\title{
图HAD
}

ISSN-L: 2530-5115

DOI: http://doi.org/10.22585/hospdomic.vli2.13

\section{Proyecto HAD 2020: una propuesta para consolidar la hospitalización a domicilio en España}

\section{HAD 2020 Project: A proposal to consolidate hospital- based home care in Spain}

Oriol Estrada Cuxart', Beatriz Massa Domínguez², Miguel Ángel Ponce González ${ }^{3}$, Manuel Mirón Rubio ${ }^{4}$, Anna Torres Corts ${ }^{5}$, Abel Mujal Martínez ${ }^{6}$, Laura Alepuz Vidal', Francisco Antón Botella ${ }^{8}$, Manuel del Río Vizoso9, Magdalena Fernández Martínez de Mandojana ${ }^{10}$, Leticia Hermida Porto ${ }^{11}$; Sociedad Española de Hospitalización a Domicilio (SEHAD)

1. Hospital Germans Trias i Pujol, Badalona, España.

2. Hospital Universitario, Sant Joan d'Alacant, España.

3. Hospital Universitario Dr. Negrín, Gran Canaria, España.

4. Hospital Universitario de Torrejón, Torrejón de Ardoz, España.

5. Hospital Dos de Maig, Barcelona, España.

6. Corporació Sanitaria Parc Taulí, Sabadell, España.

7. Hospital Comarcal Marina Baixa, La Vila Joiosa, España.

8. Hospital San Pedro, Logroño, España.

9. Hospital Son Espases, Palma, España.

10. Organización Sanitaria Integrada Bajo Deba, Mendaro, España.

11. Complexo Hospitalario Universitario, A Coruña, España.

Correspondencia/Correspondence

Dr. Oriol Estrada Cuxart

oestrada@gencat.cat

Hospital Germans Trias i Pujol, Badalona,

España.

Recibido/Received

22.11.2016

Aceptado/Accepted

29.11 .2016
Conflicto de Intereses/Competing interest Las autoras y autores de este trabajo declaran la no existencia de ningún tipo de conflicto de interés.

Financiación/Fundings

Sin financiación.

CÓMO CITAR ESTE TRABAJO | HOW TO CITE THIS PAPER

Estrada Cuxart O, Massa Domínguez B, Ponce González MA, Mirón Rubio M, Torres Corts A, Mujal Martínez A, et al. Proyecto HAD 2020: una propuesta para consolidar la hospitalización a domicilio en España. Hosp Domic. 2017;1(2):93-117. 


\section{RESUMEN}

La Hospitalización a Domicilio (HAD) apareció en España hace 36 años con la apertura de diversas unidades. El empuje inicial fue truncado por la falta de liderazgo político y en ocasiones también clínico. La realidad actual ofrece una implantación irregular con disparidad de modelos asistenciales y de recursos. La Sociedad Española de Hospitalización a Domicilio (SEHAD) no ha jugado el papel de liderazgo científico y profesional esperado. Se diseñó el «Plan HAD2020: clave de futuro» como revulsivo. Se trata de un ambicioso proyecto a 4 años para consolidar la HAD como modalidad asistencial. Su despliegue consta de cinco fases. Preparación: se establecieron las bases del plan estratégico (PE). Análisis de situación: se realizó una encuesta nacional a las 106 unidades operativas (datos 2014). Validación del PE: aportaciones y propuestas de actuación de los miembros de SEHAD. Congreso Nacional 2016: presentación y aprobación de conclusiones y propuestas del PE. Fase de despliegue PE: se extenderá hasta el año 2020 y la ejecutarán diversos equipos referentes, repartidos en cinco líneas de trabajo.

El objetivo final fijado para el año 2020 consiste en: consensuar un modelo asistencial más homogéneo; promover la formación y reconocimiento profesional de quienes trabajan en la HAD; que cada hospital de España tenga una unidad de $\mathrm{HAD}$; reconocimiento y potenciación por el sistema de salud nacional.

HAD2020 ha marcado un punto de inflexión en la SEHAD. El camino trazado y el esfuerzo de todos los profesionales HAD permitirán alcanzar aquella visión que persiguieron los pioneros de la HAD en España.

Palabras clave: Servicios de Atención a Domicilio Provisto por Hospital; Desarrollo de Programa; Gestión de la Calidad; Formulación de Proyectos

\section{ABSTRACT}

Hospital at home $(\mathrm{HAH})$ appeared in Spain 36 years ago with the opening of several units. The initial push was truncated by the lack of political leadership and sometimes clinical as well. The current reality offers an irregular implementation with a wide disparity of assistance and resource models. The Sociedad Española de Hospitalización a Domicilio (SEHAD) has not played either the expected scientific or professional leadership roles. The "Plan HAD2020: key of the future" was designed as revulsive. This is an ambitious 4-year project to consolidate $\mathrm{HAH}$ as a care modality. Its deployment consists of five phases. Preparation: the foundations of the strategic plan (EP) were established. Situation analysis: a national survey was carried out on the 106 operational units (data 2014). Validation of the EP: contributions and proposals of action of the members of SEHAD. National Congress 2016: presentation and approval of EP conclusions and proposals. EP deployment phase: it will be extended until 2020 and will be executed by various teams of referents spread over five lines of work.

The final objective set for the year 2020 is: to come up with a more homogenous care model; to promote the training and professional recognition of those who work in the HAD; that each hospital in Spain has a $\mathrm{HAH}$ unit; recognition and empowerment by the national health system.

HAD2020 has marked an inflection point in the SEHAD. The traced path and the effort of all the $\mathrm{HAH}$ professionals will allow reaching the vision which the pioneers of the $\mathrm{HAH}$ in Spain pursued.

Keywords: Home Care Services, Hospital-Based; Program Development; Quality Management; Project Formulation 


\section{INTRODUCCIÓN}

\section{Justificación del Proyecto HAD 2020}

El presente documento tiene como objeto exponer las conclusiones y logros de la fase de planteamiento y validación de un plan estratégico para el desarrollo de la Hospitalización a Domicilio (HAD) que encargó la Sociedad Española de Hospitalización a Domicilio (SEHAD) hace un año y medio. Los resultados de la primera fase de este plan se presentaron en el XIII Congreso Nacional de Hospitalización a Domicilio (Las Palmas de Gran Canaria, 21 al 23 Abril 2016) ante el Secretario General de Sanidad y Consumo (Dr. José Javier Castrodeza Sanz) y el Director General de Ordenación Sanitaria (Sr. Carlos Jesús Moreno Sánchez). En el mismo congreso los miembros de la sociedad aprobaron en sesión plenaria los planteamientos formulados en el plan estratégico para su desarrollo en los siguientes 4 años.

Para resolver satisfactoriamente los innumerables retos que plantea el futuro de la sanidad, es preciso contar con la experiencia de los profesionales que trabajan en ella. Las sociedades científicas tienen una función importante a la hora de aportar conocimiento experto y proponer soluciones innovadoras que contribuyan al avance del sistema y a su sostenibilidad. Encauzan las inquietudes de sus integrantes ya no tan solo para resolver posibles reivindicaciones corporativas sino, aún más importante, para ponerse al servicio de las instituciones públicas como aliado clave en la mejora del sistema. En esta línea la SEHAD se pone a la disposición del Ministerio y de las autoridades autonómicas para consolidar esta modalidad asistencial que aporta mejoras en calidad de la atención, seguridad del paciente, integración de los recursos y un significativo ahorro de costes respecto a la hospitalización convencional.

Hoy en día, nadie duda de que la HAD aporta ventajas como alternativa a la hospitalización convencional. Actualmente existe evidencia acerca de dichas ventajas en lo que respecta a la eficacia, seguridad y al alto nivel de satisfacción de esta modalidad asistencial. Los factores clave para su correcta implementación son garantizar una adecuada selección de los pacientes tributarios de HAD y disponer de un equipo experimentado en atención de casos complejos en el domicilio. A pesar de que no se dispone de estudios exhaustivos que lo confirmen, distintos trabajos en la literatura internacional y un número mayor de grupos nacionales que aportan sus datos de costes, parecen corroborar que a los beneficios asistenciales hay que sumar un ahorro de costes respecto a la hospitalización convencional.

¿Por qué un plan estratégico? HAD 2020 es un proyecto que nace a demanda de los socios de la SEHAD que así lo expresaron a la junta directiva durante las Jornadas del año 2015 en Santiago de Compostela. El colectivo de profesionales era consciente de que para conseguir consolidar la HAD como una opción asistencial relevante en el sistema sanitario nacional había que iniciar un trabajo de replanteamiento en profundidad, colaboración con las instituciones y de información entre políticos, profesionales y la Sociedad en general.

La SEHAD ha realizado un ejercicio de posicionamiento estratégico con HAD 2020. Conocer la situación real de esta modalidad asistencial a día de hoy en España. Identificar y analizar cuáles son los principales factores que condicionan e impiden su completo desarrollo. Conocer y aprovechar las principales palancas que pueden otorgar a la HAD el papel relevante que merece y que está llamada a desempeñar en nuestro sistema sanitario en los próximos años. Por último, pero a la vez como elemento de mayor importancia, HAD 2020 propone una agenda de trabajo para los próximos 4 años con actuaciones encaminadas a cumplir los objetivos que se plantean en su presentación. Una vez concluida la primera fase de reflexión y planteamiento estratégico, se ha iniciado la segunda fase de despliegue de las líneas de acción consensuadas en el Congreso Nacional. 


\section{Historia de la HAD en España}

La HAD aparece como modalidad asistencial en 1947 en Nueva York de la mano del Dr. Bluestone. Desde el principio se destacó la importancia del domicilio como escenario terapéutico. Frente a la tecnificación del hospital orientado a la resolución de enfermedades, es preciso tratar al enfermo conociendo el medio en el que vive. En palabras de Martin Cherkasky, responsable de la primera unidad de HAD y precursor de la medicina social, es tan importante conocer los condicionantes sociales de un enfermo (qué come, dónde vive, etc.) como el dictamen de una baciloscopia de esputo (1). Diez años más tarde la Organización Mundial de La Salud (2) fijaba su posición a favor de la extensión de las actividades hospitalarias más allá de los muros del centro. Promovía la atención especializada en el domicilio. En dicho informe se definía de la siguiente forma la asistencia domiciliaria:

«Un sistema eficaz de asistencia domiciliaria exige que el enfermo reciba en su domicilio una asistencia equivalente, en cuanto sea posible, a la proporcionada a los enfermos en un hospital».

En el mismo documento se hacía énfasis en que esta modalidad favorecía la individualización de la asistencia médica, economizaba plazas en los hospitales y resultaba menos costoso que la hospitalización convencional.

En España el desarrollo de la HAD comenzó en el año 1981 en el Hospital Provincial de Madrid (hoy Hospital General Universitario Gregorio Marañón) y posteriormente en otros grandes hospitales del estado. A pesar de que el legislador contempló en su momento la creación de unidades de HAD en la estructura de los hospitales con la OM de 1985 (3) y posterior RD de 1987 (4), junto con el reconocimiento de dicha actividad en el ámbito hospitalario en la LGS de 1986 (5), la evolución posterior del sistema sanitario nacional no consolidó este desarrollo. Únicamente el País Vasco, la Comunidad Valenciana y Galicia cuentan a día de hoy con un programa específico de HAD de ámbito poblacional y con dependencia del Servicio Autonómico de Salud. La falta de un marco regulador específico y de incentivos dirigidos a promover el desarrollo de estas unidades ha dejado a merced de la iniciativa individual de profesionales convencidos y de gerentes emprendedores la creación de unidades en algunos centros hospitalarios. Este hecho supuso el declive e incluso el cierre de muchas de las unidades pioneras en España y la aparición de unidades sin un modelo asistencial definido, ni una estructura de recursos adecuada o tan solo una cartera de servicios homogénea. Un factor no menor que ha contribuido al escaso desarrollo de la HAD en el estado ha sido la falta de un modelo de pago específico. La situación de la HAD contrasta con otras modalidades emergentes en su día, como el hospital de día, los centros de diálisis o, caso paradigmático, la cirugía mayor ambulatoria. Todas ellas están ampliamente consolidadas en todos los centros y en todas ellas el liderazgo en planificación y despliegue ha sido evidente por parte de las instituciones públicas. A día de hoy nadie discute su utilidad.

A pesar de este desarrollo irregular cabe destacar que la HAD aparece como una de las prestaciones reconocidas en la Cartera de Servicios Comunes del SNS de 2006 (6). Recientemente se ha publicado un RD (7) que regula el registro de la actividad en atención especializada que incluye específicamente la HAD como actividad propia, por lo que con la aplicación del citado Registro a principios de 2018 en los centros públicos, será necesaria la recogida de datos específicos de HAD en todos los centros del SNS. 


\section{Situación actual de la HAD en España y factores limitantes para su desarrollo}

El año 2006 se fundó la SEHAD por la fusión de diversas sociedades autonómicas. Esta sociedad ha aglutinado a los profesionales que se dedican a la HAD en España. Actualmente se cuentan en activo 111 unidades. Su distribución geográfica es irregular. Castilla La Mancha no tiene unidades de HAD. En Aragón su despliegue es incipiente y en Andalucía únicamente se cuentan 5 unidades, siendo la comunidad con mayor población del estado. En la figura 1 se presenta la distribución de unidades HAD por Comunidades Autónomas.

Figura 1. Distribución de unidades Hospitalización a Domicilio por Comunidades Autónomas de España.

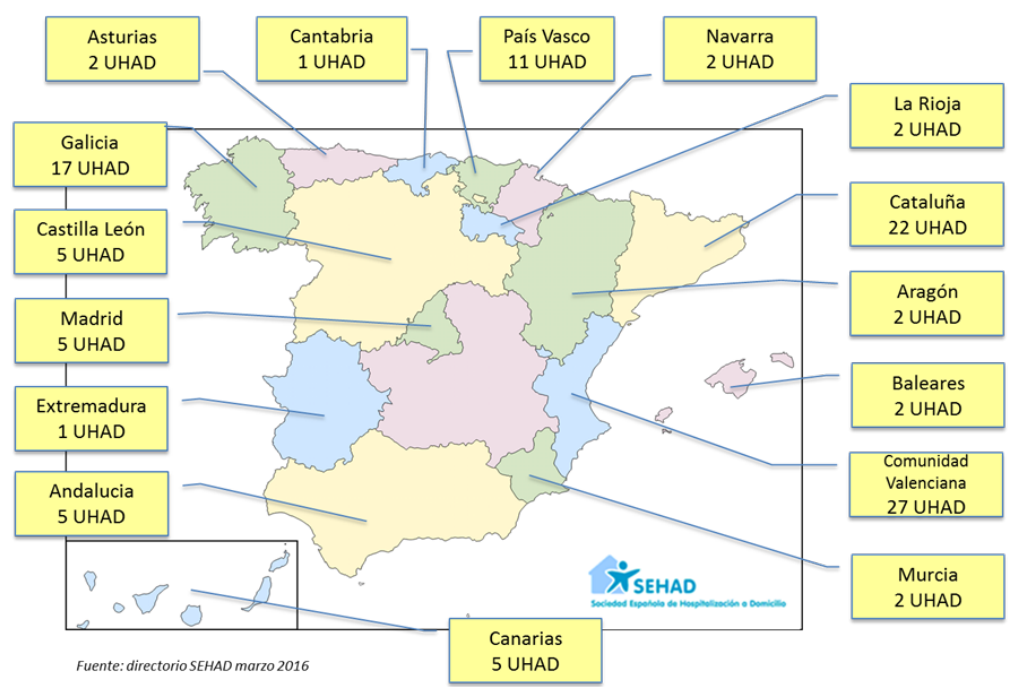

En ausencia de indicaciones específicas sobre qué procesos son tributarios de HAD, los recursos mínimos necesarios o de indicadores específicos de seguimiento, el panorama actual muestra una gran disparidad entre las diversas unidades en lo que respecta a oferta asistencial, complejidad de la casuística atendida, de los recursos disponibles y del número y titulación de los profesionales al cargo. La utilización del CMBD de hospitalización convencional y el uso de los DRG no son adecuados para monitorizar la actividad y los resultados de la HAD.

En resumen, cabe destacar como factores que han lastrado el desarrollo de la HAD en España los que se enumeran a continuación (8):

- Falta de un impulso institucional a nivel estatal.

- Gran heterogeneidad de las unidades y de su distribución geográfica.

- Disparidad de oferta asistencial y de cartera de servicios.

- Disparidad de recursos entre las distintas unidades.

- Ausencia de un sistema de pago específico para la HAD en la mayoría de CCAA.

- Falta de reconocimiento profesional. 


\section{DESARROLLO DE LA PRIMERA FASE DEL PROYECTO HAD 2020}

En el año 2015 la Junta Directiva de la SEHAD designó un grupo de 10 expertos, médicos y enfermeras, de diferentes CCAA y que pertenecían a unidades de diversos tamaños y nivel de complejidad en la atención. Todos ellos fueron invitados a participar en el proyecto como referentes de la Sociedad por su alto conocimiento e implicación en la HAD. Esta primera fase de preparación contaba con tres grandes ejes de actuación.

Por un lado conocer la realidad de la HAD en España en el momento actual. En base a las conclusiones preliminares elaboradas por el grupo de expertos, se ha diseñado y lanzado una encuesta dirigida a todas las unidades HAD en activo con la intención de conocer directamente la realidad de estas unidades.

De forma paralela, se ha construido el diagnóstico estratégico de situación. Se ha analizado en profundidad la situación estratégica de la HAD en España desde diversos puntos de vista. Durante este período se ha contado con la colaboración de profesionales y expertos externos que han aportado su perspectiva de la HAD. Para validar estas conclusiones se desplegó un cuestionario que respondió un amplio número de profesionales HAD. Finalmente el diagnóstico estratégico de situación planteado y realizado ha contado con la participación de unos 140 profesionales HAD que han aportado su visión.

En tercer lugar y como elemento más importante del trabajo de posicionamiento estratégico se hizo una propuesta de líneas de actuación diseñadas con objeto de dar respuesta a los elementos de la visión estratégica del HAD2020. La ponencia de propuesta fue sometida al escrutinio y valoración de los miembros de SEHAD y finalmente validada durante el Congreso Nacional de 2016.

\section{Situación actual de la HAD en España: Encuesta nacional de unidades HAD}

Se implicó a los responsables de las unidades HAD en activo. El registro de unidades de SEHAD constaba en marzo de 2016 de 110 unidades. Respondieron 78 unidades (71\%). Las dimensiones principales a los que hacía referencia la encuesta eran:

- Organización de las unidades

- Recursos humanos

- Recursos materiales, tecnológicos e infraestructura de cada una de ellas

- Sistema de información

- Cartera de servicios, actividad asistencial y resultados durante el año 2014

- Docencia e investigación

- Modelo asistencial (circuitos, protocolos, guías clínicas, etc.)

Esta muestra representativa y detallada de aspectos que evidencian la realidad de la HAD en España supone un excelente punto de partida para identificar los ámbitos de mejora en los próximos años (9). A modo de resumen, como datos más relevantes de esta encuesta se destacan los siguientes:

- Unidades de reciente creación (<5 años): 9,6\%.

- Unidades HAD con cobertura poblacional de su área de referencia: $48,7 \%$.

- Ratio poblacional de plazas HAD: 0,08/103 habitantes.

- Altas HAD: media por unidad 866 (DE 634).

- Unidades pequeñas por su capacidad asistencial desplegada (<30 plazas): 42,3\%.

- Unidades que disponen de información completa de costes: 20,6\%. 
- Personal adscrito a las unidades participantes en la encuesta nacional HAD: 867 profesionales (30,3\% médicos, 57,7\% enfermeras, otros $12 \%$ ).

- Ratio profesionales media de las 78 unidades que respondieron: 0,40/plaza HAD (H Convencional 1,86/cama).

- Unidades que practican el ingreso en régimen de voluntariedad de pacientes y familiares: $100 \%$.

- Unidades que no disponen de ningún dispositivo propio de atención continuada: 26,9\%.

- Unidades que tienen definidos circuitos de continuidad asistencial: 56,3\%.

- Unidades que registran actividad docente: pregrado 65\%; postgrado $77 \%$.

- Unidades con actividad de formación continuada específica: 32,9\%.

- Unidades con actividad investigadora (participación en publicaciones indexadas): 25,6\%.

\section{Análisis estratégico de situación. Cuestionario de validación a miembros de SEHAD}

Para completar el segundo objetivo de la fase de preparación, se implementó una línea de acción que consistió en difundir un cuestionario de validación de las conclusiones del grupo de trabajo, dirigido a todos los profesionales de la HAD. Respondieron 136 profesionales (un 40\% de los socios de la SEHAD). De las respuestas obtenidas se priorizaron las líneas de actuación estratégica para los próximos 4 años.

Finalmente todo este trabajo se difundió mediante un plan de comunicación con presencia en medios de comunicación generalistas y del sector sanitario. Se informó a los participantes en el Congreso Nacional en una sesión específica en la que los asistentes validaron la estrategia propuesta por la Sociedad para los próximos 4 años.

A continuación se detallan las conclusiones más relevantes del diagnóstico compartido:

\section{Descripción de la visión estratégica de la HAD en España en el horizonte 2020.}

VISIÓN 2020: LA HAD será una modalidad relevante del sistema sanitario español. De implantación prácticamente universal, ofrecerá la más favorable oportunidad de atención especializada en el domicilio del paciente con los mejores resultados en eficacia clínica, seguridad y satisfacción para el propio paciente y sus familiares. Será un elemento clave favorecedor de la sostenibilidad de la atención hospitalaria.

EXPLICACIÓN: Las unidades de HAD están llamadas a ser servicios/unidades multidisciplinarias independientes, con gestión autónoma y eficiente que unificarán prácticas clínicas basadas en la evidencia de forma excelente para atender al paciente en el domicilio como alternativa a la hospitalización convencional de procesos agudos, agudizaciones de procesos crónicos o pacientes paliativos complejos. El auge de esta modalidad de atención favorecerá la coordinación con el resto de niveles asistenciales, garantizando el seguimiento y la atención del paciente en fase de estabilidad clínica. La HAD, con un modelo asistencial definido y reconocido, sustituirá una parte significativa de las hospitalizaciones convencionales de cada centro, atendiendo a su capacidad de tratar a enfermos complejos con resultados no inferiores a la hospitalización convencional. El ahorro de costes generado deberá reinvertirse en adaptar el sistema sanitario a sus nuevos retos. Por mediación de las sociedades científicas u otros grupos colaborativos, la HAD generará conocimiento y evidencia propia que permitirá una mejor formación de los profesionales y seguir avanzando en su consolidación. 


\section{Elementos constituyentes de la visión estratégica.}

La visión futura de HAD se compone de una serie de elementos alrededor de los cuales se centrará el trabajo en los próximos años. En la tabla 1 se explican dichos elementos.

Tabla 1. Elementos constituyentes de la visión estratégica del HAD 2020

\begin{tabular}{|c|c|c|}
\hline ÁMBITO & OBJETIVOS & VISIÓN HAD 2020 \\
\hline \multirow[t]{5}{*}{$\begin{array}{l}\text { Modelo } \\
\text { Asistencial }\end{array}$} & $\begin{array}{l}\text { Modelo asistencial } \\
\text { definido }\end{array}$ & $\begin{array}{l}\text { Homogeneidad, mínimos comunes a todas las unidades } \\
\text { (recursos y actividad). Cartera básica común }\end{array}$ \\
\hline & Atención integrada & $\begin{array}{l}\text { Integración funcional con otros servicios y niveles } \\
\text { (MI,HDIA,UCIAS, AP) y alternativas a la hospitalización }\end{array}$ \\
\hline & $\begin{array}{l}\text { Modelo de } \\
\text { acreditación } \\
\text { propio. }\end{array}$ & $\begin{array}{l}\text { Generar una dinámica de acreditación de las unidades basada } \\
\text { en el modelo asistencial, los resultados clínicos, la seguridad del } \\
\text { paciente, la calidad de la atención y la gestión de los recursos }\end{array}$ \\
\hline & $\begin{array}{l}\text { Uso intensivo } \\
\text { de nuevas } \\
\text { tecnologías }\end{array}$ & $\begin{array}{l}\text { Aplicar nuevas tecnologías en facilitar el control del paciente en } \\
\text { su domicilio }\end{array}$ \\
\hline & $\begin{array}{l}\text { Cobertura } \\
\text { ampliada }\end{array}$ & $\begin{array}{l}\text { Cobertura } 365 \text { días al año. Garantizando dispositivo atención } \\
\text { continuada }\end{array}$ \\
\hline \multirow[t]{3}{*}{ Recursos HAD } & $\begin{array}{l}\text { Universalidad de } \\
\text { su implantación, }\end{array}$ & $\begin{array}{l}\text { Todos los hospitales deben tener una HAD. Opción de } \\
\text { hospitalización "por defecto". Accesible para toda la población }\end{array}$ \\
\hline & $\begin{array}{l}\text { Sistemas de } \\
\text { información }\end{array}$ & $\begin{array}{l}\text { Homologables y adaptados. Información sobre gestión de } \\
\text { costes. SI específicos para HAD (indicadores, Bases de datos } \\
\text { comunes) }\end{array}$ \\
\hline & $\begin{array}{l}\text { Autonomía de } \\
\text { gestión }\end{array}$ & $\begin{array}{l}\text { Dotar a la HAD de capacidad de gestión. Consideración de } \\
\text { unidades de gestión clínica }\end{array}$ \\
\hline $\begin{array}{l}\text { Perfil } \\
\text { profesional }\end{array}$ & $\begin{array}{l}\text { Reconocimiento } \\
\text { profesional }\end{array}$ & $\begin{array}{l}\text { Mejorar la categoría profesional (formación continuada } \\
\text { específica, docencia en pre/postgrado) }\end{array}$ \\
\hline $\begin{array}{l}\text { Promover la } \\
\text { investigación }\end{array}$ & $\begin{array}{l}\text { Gestión } \\
\text { conocimiento. } \\
\text { Investigación } \\
\text { clínica. }\end{array}$ & $\begin{array}{l}\text { Capacidad para generar conocimiento y evidencia sobre la HAD } \\
\text { Publicar experiencias. Practicar y fomentar el Benchmarking }\end{array}$ \\
\hline Sostenibilidad & $\begin{array}{l}\text { Financiación } \\
\text { específica }\end{array}$ & $\begin{array}{l}\text { Sostenibilidad. Modelo de financiación HAD justo. Ajustado } \\
\text { por dispersión, actividad, complejidad y resultados. Modelo } \\
\text { incluido en la administración pública y reconocido como línea de } \\
\text { facturación de los hospitales }\end{array}$ \\
\hline Impacto social & $\begin{array}{l}\text { Prestigio social de } \\
\text { la HAD }\end{array}$ & $\begin{array}{l}\text { Impacto en el sistema y en los medios. Imagen de excelencia. } \\
\text { Buscar una revista de referencia de la sociedad científica }\end{array}$ \\
\hline
\end{tabular}




\section{Matriz DAFO de diagnóstico estratégico de la HAD en España.}

Se construyó la matriz DAFO concerniente al momento actual de la HAD. Para ello, inicialmente el panel de expertos analizó los principales factores determinantes de la situación actual de la HAD en España. Se revisaron los principales condicionantes internos identificando fortalezas y debilidades. Los determinantes externos se agruparon en oportunidades y en amenazas. Los resultados se agruparon mostrándose el número de votos que registró cada una de las opciones identificadas. Ver tabla 2.

Tabla 2. Matriz DAFO de diagnóstico estratégico de la HAD en España*

\begin{tabular}{|c|c|c|c|}
\hline FORTALEZAS & DEBILIDADES & OPORTUNIDADES & AMENAZAS \\
\hline $\begin{array}{l}\text { Implicación con } \\
\text { la asistencia. } \\
\text { Identificación con la } \\
\text { HAD [7] }\end{array}$ & $\begin{array}{l}\text { Variabilidad en la } \\
\text { atención entre unidades } \\
\text { [9] }\end{array}$ & $\begin{array}{l}\text { Desarrollo de nuevas } \\
\text { TIC y sistemas de } \\
\text { información [9] }\end{array}$ & $\begin{array}{l}\text { Percepción como } \\
\text { amenaza por parte de } \\
\text { AP y algunos servicios } \\
\text { hospitalarios [9] }\end{array}$ \\
\hline $\begin{array}{l}\text { Trabajo en equipo } \\
\text { multidisciplinario [7] }\end{array}$ & $\begin{array}{l}\text { Financiación insuficiente } \\
\text { o inexistente [8] }\end{array}$ & $\begin{array}{l}\text { Atención más } \\
\text { centrada en procesos } \\
\text { y rutas asistenciales. } \\
\text { Oportunidad de } \\
\text { liderazgo para la HAD } \\
\text { [9] }\end{array}$ & $\begin{array}{l}\text { Indiferencia, } \\
\text { desconocimiento de } \\
\text { políticos y planificadores } \\
\text { [8] }\end{array}$ \\
\hline $\begin{array}{l}\text { Buen ambiente laboral } \\
\text { [6] }\end{array}$ & $\begin{array}{l}\text { Disparidad de carteras } \\
\text { de servicios. ¿Qué es? y } \\
\text { ¿Qué no es?[7] }\end{array}$ & $\begin{array}{l}\text { Necesidad de potenciar } \\
\text { continuum asistencial } \\
\text { entre el hospital y la } \\
\text { atención primaria [rol } \\
\text { HAD] [6] }\end{array}$ & $\begin{array}{l}\text { Fragmentación del } \\
\text { proceso asistencial. No } \\
\text { visión integral [7] }\end{array}$ \\
\hline $\begin{array}{l}\text { Atención de calidad } \\
\text { centrada en el paciente } \\
\text { [6] }\end{array}$ & $\begin{array}{l}\text { Falta de indicadores } \\
\text { específicos. No se } \\
\text { pueden conocer } \\
\text { resultados ni } \\
\text { compararlos [6] }\end{array}$ & $\begin{array}{l}\text { Salir de la crisis: } \\
\text { búsqueda de } \\
\text { organizaciones eficientes } \\
\text { y con buenos resultados } \\
\text { clínicos, HAD en la línea } \\
\text { si genera evidencia } \\
\text { propia [6] }\end{array}$ & $\begin{array}{l}\text { Falta de definición en } \\
\text { procesos transversales. } \\
\text { Liderazgos externos en } \\
\text { pacientes crónicos [6] }\end{array}$ \\
\hline $\begin{array}{l}\text { Se percibe cierta } \\
\text { dinámica de } \\
\text { modernización y } \\
\text { cambio en la HAD [5] }\end{array}$ & $\begin{array}{l}\text { Poco reconocimiento } \\
\text { profesional. Problema de } \\
\text { "autoestima" [6] }\end{array}$ & $\begin{array}{l}\text { Intensificar la relación } \\
\text { con otras sociedades } \\
\text { científicas [5] }\end{array}$ & $\begin{array}{l}\text { Dirección estratégica } \\
\text { en salud errática y } \\
\text { politizada [5] }\end{array}$ \\
\hline $\begin{array}{l}\text { Alto nivel de } \\
\text { satisfacción del } \\
\text { paciente y su familia [4] }\end{array}$ & $\begin{array}{l}\text { Investigación HAD, } \\
\text { testimonial. No se genera } \\
\text { evidencia [6] }\end{array}$ & $\begin{array}{l}\text { Oportunidad de trabajar } \\
\text { y compartir datos en } \\
\text { red entre las UHAD del } \\
\text { estado [4] }\end{array}$ & $\begin{array}{l}\text { Falta de interés de } \\
\text { los clientes internos } \\
\text { [Servicios] en la HAD [5] }\end{array}$ \\
\hline $\begin{array}{l}\text { Flexibilidad y } \\
\text { capacidad de } \\
\text { adaptación [3] }\end{array}$ & $\begin{array}{l}\text { Falta un programa } \\
\text { estatal que reconozca y } \\
\text { consolide la HAD [5] }\end{array}$ & $\begin{array}{l}\text { Proponer y desarrollar un } \\
\text { plan nacional HAD [4] }\end{array}$ & $\begin{array}{l}\text { Gerentes que busquen } \\
\text { oportunidad de ahorro } \\
\text { a corto plazo. Recortar } \\
\text { programas HAD para } \\
\text { ahorrar recursos [4] }\end{array}$ \\
\hline
\end{tabular}




\begin{tabular}{|c|c|c|c|}
\hline $\begin{array}{l}\text { Perfil resolutivo para el } \\
\text { hospital. Cubre déficits } \\
\text { asistenciales [3] }\end{array}$ & $\begin{array}{l}\text { Falta formación } \\
\text { específica [5] }\end{array}$ & $\begin{array}{l}\text { Utilizar la gestión clínica } \\
\text { como herramienta de } \\
\text { cambio y de mejora [4] }\end{array}$ & $\begin{array}{l}\text { Falta de liderazgo } \\
\text { directivo HAD en el } \\
\text { centro por parte del } \\
\text { director médico y del } \\
\text { gerente [4] }\end{array}$ \\
\hline $\begin{array}{l}\text { Profesionales } \\
\text { experimentados. } \\
\text { Protocolos existentes } \\
\text { [2] }\end{array}$ & $\begin{array}{l}\text { Disparidad de recursos. } \\
\text { En general faltan [5] }\end{array}$ & $\begin{array}{l}\text { Buena aceptación en } \\
\text { algunas publicaciones } \\
\text { de trabajos de } \\
\text { investigación clínica en } \\
\text { HAD [3] }\end{array}$ & $\begin{array}{l}\text { Priorizar financiación de } \\
\text { la complejidad frente } \\
\text { a alternativas como la } \\
\text { HAD [4] }\end{array}$ \\
\hline $\begin{array}{l}\text { Rol de nexo asistencial } \\
\text { entre el hospital y la } \\
\text { atención primaria [2] }\end{array}$ & $\begin{array}{l}\text { Irrelevancia de la } \\
\text { SEHAD. Solo organiza } \\
\text { congreso [5] }\end{array}$ & $\begin{array}{l}\text { SEHAD como } \\
\text { aglutinador y parte } \\
\text { frente a políticos y } \\
\text { planificadores [3] }\end{array}$ & $\begin{array}{l}\text { Fragmentación del mapa } \\
\text { sanitario español. } 17 \\
\text { realidades distintas [3] }\end{array}$ \\
\hline $\begin{array}{l}\text { Amplio horario de } \\
\text { atención }\end{array}$ & $\begin{array}{l}\text { Utilización irregular de } \\
\text { las nuevas tecnologías, } \\
\text { más bien pobre [5] }\end{array}$ & $\begin{array}{l}\text { Buena predisposición } \\
\text { de algunos agentes } \\
\text { externos como socios de } \\
\text { la HAD: industria [2] }\end{array}$ & $\begin{array}{l}\text { Competencia con } \\
\text { ATDOM de atención } \\
\text { primaria [3] }\end{array}$ \\
\hline $\begin{array}{l}\text { Capacidad de alejar al } \\
\text { paciente del hospital } \\
\text { y mantenerlo en su } \\
\text { entorno }\end{array}$ & $\begin{array}{l}\text { No se conocen datos } \\
\text { concluyentes de costes. } \\
\text { No se puede afirmar la } \\
\text { eficiencia [4] }\end{array}$ & $\begin{array}{l}\text { Sobrecarga asistencial } \\
\text { de atención primaria. } \\
\text { Papel de la HAD menos } \\
\text { discutido [1] }\end{array}$ & $\begin{array}{l}\text { Presión asistencial } \\
\text { según necesidades de } \\
\text { los Servicios. Demanda } \\
\text { no controlable [3] }\end{array}$ \\
\hline \multirow[t]{6}{*}{$\begin{array}{l}\text { Implicación con } \\
\text { la asistencia. } \\
\text { Identificación con la } \\
\text { HAD [7] }\end{array}$} & $\begin{array}{l}\text { Escaso impacto en los } \\
\text { medios y en la sociedad } \\
{[4]}\end{array}$ & & $\begin{array}{l}\text { Descoordinación con los } \\
\text { recursos socio-sanitarios } \\
\text { [3] }\end{array}$ \\
\hline & $\begin{array}{l}\text { No se visualiza como } \\
\text { alternativa a la HC. Es un } \\
\text { complemento [3] }\end{array}$ & & $\begin{array}{l}\text { Competencia en } \\
\text { investigación. No acceso } \\
\text { a fondos competitivos [2] }\end{array}$ \\
\hline & $\begin{array}{l}\text { Pendiente de la } \\
\text { fidelización de algunos } \\
\text { servicios [3] }\end{array}$ & & $\begin{array}{l}\text { Falta de cultura y } \\
\text { concienciación sanitaria } \\
\text { de la población general } \\
\text { [1] }\end{array}$ \\
\hline & $\begin{array}{l}\text { Falta cultura de } \\
\text { evaluación y mejora } \\
\text { continua [2] }\end{array}$ & & \\
\hline & $\begin{array}{l}\text { En bastantes centros aún } \\
\text { no se dispone de } \mathrm{H}^{\mathrm{a}} \mathrm{C}^{\mathrm{a}} \\
\text { informatizada [2] }\end{array}$ & & \\
\hline & $\begin{array}{l}\text { Descoordinación con } \\
\text { atención socio-sanitaria } \\
\text { [1] }\end{array}$ & & \\
\hline \multicolumn{4}{|c|}{$\begin{array}{l}\text { * El número entre corchetes expresa la relevancia de cada propuesta en el conjunto, siendo } 9 \text { la máxima } \\
\text { puntuación y } 1 \text { la mínima. }\end{array}$} \\
\hline
\end{tabular}




\section{Líneas estratégicas de actuación}

El tercer elemento de este análisis estratégico consistió en la definición de una propuesta de actuación que diera respuesta a los diferentes elementos significados en el Plan HAD 2020. Una vez construida esta propuesta se sometió igualmente a la validación de los miembros de la asamblea de la SEHAD. Finalmente las líneas de acción que recibieron mayor apoyo de los miembros SEHAD que participaron en su validación se describen a continuación por orden de relevancia.

\section{Definición de líneas de actuación estratégica}

La propuesta validada consiste en definir unas vías de actuación estratégica encaminadas a conseguir nuestra visión, apoyándonos en nuestras fortalezas internas, mejorando nuestras debilidades, evitando las amenazas externas y aprovechando las oportunidades. Las vías de actuación estratégica son grandes ámbitos de actuación donde se definirán objetivos estratégicos concretos basados en medidas a tomar, proyectos a desarrollar, etc. Estas líneas han sido aprobadas por los miembros de SEHAD y serán los respectivos grupos de trabajo los que definan los objetivos y plazos para completar la tarea propuesta.

\section{- Modelo asistencial común}

a. El objetivo será coordinar un trabajo que genere el más amplio consenso en la definición del concepto de HAD, del Catálogo de actividades y la Cartera de Servicios que le son propias. Así como de revisar el proceso asistencial.

b. Plan de calidad. En una segunda etapa este proyecto debe llevar aparejada la búsqueda de un sistema de acreditación de las unidades en función de los estándares objetivados y consensuados previamente.

c. En una tercera fase promover la disminución de la variabilidad clínica. Identificar procesos asistenciales para elaborar/actualizar guías clínicas propias (Libro de Procedimientos) o con otras sociedades.

\section{- Sistema de información compartido}

a. Definir y consensuar los principales indicadores específicos del cuadro de mandos (CM) de la HAD.

b. Diseñar y poner en producción un CM específico.

c. Creación de la norma de los indicadores establecidos.

d. Uso del CM por las unidades adheridas a SEHAD. Promover el Benchmark.

e. Modelo de clasificación de pacientes HAD.

f. CMBD específico.

g. Creación de un banco de datos multicéntrico.

\section{- Promoción de la investigación}

a. Promover la creación de estudios multicéntricos en investigación clínica y sostenibilidad. Definir campos prioritarios.

b. Promover la formación en investigación, la creación de grupos colaborativos y el acceso a proyectos financiados. 


\section{- Difusión a los decisores (profesionales y políticos)}

a. Libro blanco de la HAD: estándares y Recomendaciones sobre la HAD.

b. Definición de objetivos para la difusión HAD. Políticos, planificadores, presidentes de sociedades científicas, asociaciones de enfermos, periodistas.

c. Difusión entre los profesionales de la HAD.

d. Crear un grupo de comunicación para desarrollar los objetivos anteriores, elaborar contenidos y facilitar su divulgación interna y externa.

e. Revista especializada. Fidelizar la línea editorial de alguna revista con la publicación de trabajos sobre HAD.

\section{- Profesionales sanitarios y formación HAD}

a. Definir y desarrollar plan de formación HAD específico para profesionales (médicos, enfermeras. otros) desde la SEHAD. Acreditado y con valor curricular (postgrado, máster, etc.).

b. Desarrollar competencias y habilidades de los profesionales HAD mediante formación.

c. Promover el reconocimiento de una categoría profesional propia.

d. Estudiar y comparar cargas de trabajo ajustadas. Productividad.

\section{- Modelo de financiación de la HAD}

a. Definir y aportar información sobre costes y actividad de diversas unidades.

b. Definir Escala de Unidades relativas de coste.

c. Análisis y comparación de modelos de financiación.

d. Promover iniciativas que permitan generar evidencia sobre la sostenibilidad de la HAD.

\section{Cómo puede la HAD contribuir a la mejora y sostenibilidad del modelo sanitario}

Los responsables del proyecto HAD2020 redactaron asimismo un argumentario en apoyo de la HAD como alternativa emergente. Los puntos de consenso en favor de la HAD aprobados por la SEHAD se resumen a continuación. Entre las ventajas que aporta la HAD como modalidad asistencial al paciente y al conjunto del sistema sanitario se destacan las siguientes:

1. Humaniza la atención. Aporta como elemento de valor el escenario terapéutico idóneo: el domicilio. Permite un trato personalizado y directo con el paciente y su familia. Adapta la atención a la realidad de cada enfermo.

2. Presta una atención centrada en el paciente. Este deja de ser un sujeto pasivo. Fomenta el autocuidado y empodera a los familiares y cuidadores en la atención que se le dispensa. Da mayor autonomía y seguridad en el manejo del paciente crónico complejo en el domicilio. Implica y forma al enfermo y a sus cuidadores para la etapa posterior al alta.

3. Proporciona intensidad de cuidados. Dispone de una capacidad de intervención elevada, de rango hospitalario. Permite acortar o incluso evitar el ingreso en hospitalización convencional.

4. Fomenta la continuidad asistencial. Su ámbito de actuación está cercano a la atención comunitaria, pero no invade su área de competencia. Favorece la coordinación con atención primaria así 
como la respuesta a las necesidades de la misma en pacientes que requieran cuidados complejos y sean tributarios de la HAD evitando así la institucionalización del paciente.

5. Proporciona atención multidisciplinar. La organización del trabajo precisa de un trabajo coordinado de diversos perfiles profesionales. La multidisciplinariedad de sus integrantes y su polivalencia, son valores necesarios para desarrollar un modelo de atención experto, próximo y de calidad. Supera la rigidez de servicios médicos jerarquizados del hospital y da una mejor respuesta a la diversidad de requerimientos asistenciales que se le plantean.

6. Es segura para el paciente. Por la naturaleza de su organización, los indicadores de seguridad clínica del paciente son equiparables, e incluso mejores que el de las unidades de hospitalización convencional. Tiende a evitar sobreindicación de tratamientos y de pruebas diagnósticas. Disminuye la iatrogenia.

7. Es efectiva. Los resultados obtenidos tras la atención de pacientes correctamente seleccionados son como mínimo, no inferiores a los resultados obtenidos en HC. Hay que poner de relieve la importancia en disponer de equipos expertos con una cartera de servicios homogénea y una disponibilidad y utilización de recursos estandarizada.

8. Es satisfactoria. Es una modalidad con un alto contenido de relación con el enfermo y su entorno. Este lo percibe como un elemento de calidad y atención excelente. Las unidades de HAD en España que realizan encuestas de satisfacción a sus pacientes suelen obtener resultados más elevados que la media de unidades de su centro. El índice de fidelización suele ser próximo al 100\% en los casos en que se registra.

9. Es eficiente. Su coste por estancia es sensiblemente inferior a la modalidad de HC. En aquellos enfermos tributarios de ser atendidos en régimen de $\mathrm{HAD}$, el ahorro dilapidado al mantener la $\mathrm{HC}$ se sitúa entre el 15 y el $80 \%$ de los gastos de hospitalización, según centros y patologías

10. Se adapta a los cambios. Su versatilidad propicia que sea un servicio flexible. Tiene capacidad para atender rápidamente una realidad cambiante según las necesidades de cada centro. Es un auténtico servicio asistencial de soporte transversal a todas las áreas de un centro hospitalario (médicas, quirúrgicas, gabinetes, intervencionismo, etc.).

\section{DESARROLLO DE LA SEGUNDA FASE PROYECTO HAD 2020. DESPLIEGUE Y EJECUCIÓN DE LAS LÍNEAS ESTRATÉGICAS}

Desde el mes de junio de 2016 se ha iniciado el despliegue de las distintas líneas de actuación con las tareas identificadas y la temporalización de los objetivos definidos para cada una de ellas. Este esfuerzo de toda la Sociedad tiene como objeto consolidar la HAD estandarizando su organización y actividad, potenciando su despliegue en todos los centros hospitalarios de patología aguda y promoviendo la generación de conocimiento y evidencia científica siguiendo el ejemplo de grupos de investigación clínica aplicada como el Registro TADE para tratamiento antimicrobiano intravenoso en el domicilio que ya se ha convertido en un referente internacional para esta línea de investigación. 


\title{
Línea 1: modelo asistencial común
}

\author{
Modelo asistencial de HAD: el hospital del futuro
}

El modelo centrado en el hospital tradicional cómo núcleo de la asistencia sanitaria desarrollado en España entre los 50 y los 90 ha creado grandes hospitales sobredimensionados que generan costes muy elevados. Además el entorno hospitalario representa un riesgo para el paciente por ser fuente de infecciones nosocomiales, gérmenes multirresistentes y encarnizamientos terapéuticos con excesiva realización de pruebas complementarias e ingresos innecesarios en un intento por justificar las grandes inversiones en infraestructuras hospitalarias.

Este modelo de atención centrada en el hospital tampoco ha resultado efectivo en la actual realidad social caracterizada por un progresivo envejecimiento de la población y en la que la estratificación de los usuarios muestra un número creciente de pacientes de alta complejidad (ejemplificado en el modelo asistencial de la Pirámide de Kaiser-Permanente), que consumen hasta el $80 \%$ del gasto sanitario en el anciano frágil pluripatológico por reingresos hospitalarios y visitas recurrentes a urgencias.

Para responder a los nuevos desafíos de la atención sanitaria es necesario adaptar la prestación de servicios asistenciales hacia modelos más coste-efectivos a partir de estructuras sencillas, ágiles y flexibles que potencien el tratamiento y seguimiento del paciente en su entorno. La intensidad de las actuaciones debe ser adecuada a las necesidades de los pacientes en cada momento asegurando la calidad y seguridad de los servicios prestados (Intensidad de Atención Primaria, Intensidad HAD, Intensidad de Centro Socio Sanitario, Intensidad de Hospitalización Convencional). Así, se debería favorecer la atención de los pacientes en el entorno comunitario potenciando los servicios alternativos a la hospitalización convencional.

A su vez, el rediseño en los modelos de prestación de servicios de salud exige de una comunicación fluida entre los niveles de atención para asegurar la continuidad de los cuidados del modo más confortable para el paciente y de la manera más segura y costo efectiva.

En este contexto, la HAD se perfila como un elemento vertebrador en el modelo asistencial del futuro. Sin embargo, y a pesar de las ventajas y las perspectivas de contribución a la mejora y sostenibilidad del sistema sanitario, este modelo asistencial se ha venido desarrollando en la mayoría de CCAA de una manera desestructurada, sin planificación y sin un marco regulatorio que permita alcanzar los estándares de eficiencia deseados.

El proyecto HAD 2020 pretende sentar las bases para el desarrollo de un modelo común de HAD en el estado español.

\section{Objetivos}

- Coordinar un trabajo que genere el más amplio consenso en la definición del concepto de HAD, del Catálogo de actividades y la Cartera de Servicios que le son propias, para desarrollar un modelo de gestión de mejora continua, así como revisar el proceso asistencial. Es decir, homogeneizar la definición de la cartera de servicios y de recursos necesarios. Participar en la redacción de un libro de estándares y recomendaciones para la progresiva homogeneización de las actividades comprendidas en la HAD en España. Este consenso de definiciones es urgente antes que los hospitales públicos empiecen a codificar episodios de HAD sin tener una norma a nivel estatal.

- Plan de calidad. Desarrollar un sistema de acreditación de las unidades en función de los estándares objetivados y consensuados previamente, con inclusión de las HAD en índices nacionales de valoración de la calidad asistencial en salud (EPINE, ICAR, IRAR...). 
- Promover la disminución de la variabilidad clínica. Identificar procesos asistenciales para elaborar/actualizar guías clínicas propias (Libro de Procedimientos) o con otras sociedades.

- Establecer un diccionario de términos normalizados específicos de la HAD para facilitar la generación de un modelo consensuado.

\section{Unidad de gestión clínica}

Las unidades de HAD son una gran oportunidad para desarrollar las Unidades de Gestión clínica, suponen una nueva fórmula organizativa que incorpora la cultura de la corresponsabilidad de los profesionales en la gestión de los recursos públicos y la descentralización de la gestión, orientando la actividad hacia los resultados en salud, el control de los costes sanitarios y la mayor eficacia.

El responsable de la UGC HAD debe estar claramente identificado y accesible telefónicamente con los centros de atención primaria, con objeto de garantizar la coordinación y programación desde el hospital de ingresos, consultas, estudios o actividades cooperativas.

Además la relación de la HAD con la unidad o servicio de urgencias debe ser muy clara contando preferentemente con la participación de un médico de ese servicio. El objetivo es evitar un uso inadecuado del mismo por los pacientes pluripatológicos y, en la medida de lo posible, minimizar los episodios de atención evitables en dicho servicio cuando los pacientes sufren reagudizaciones. También debe tener una relación fluida con los otros servicios hospitalarios, AP y servicios de soporte cuando no sea posible mantener por motivo social el ingreso en el domicilio como CSS.

La estructura de la HAD tiene la capacidad de adaptarse a la estructura del hospital y servicio de atención primaria correspondiente según su despliegue en cada territorio.

\section{Objetivo}

- Transformar las HAD en unidades de gestión clínica autónomas.

\section{Línea 2: sistema de información compartido}

\section{Sistemas de información}

El sistema de información se refiere al conjunto de aplicaciones informáticas que se utilizan para recoger, almacenar, gestionar y trasmitir la información de los pacientes, para que los profesionales sanitarios, los gestores y el resto del personal de la organización realicen su trabajo de manera eficaz y eficiente. Al gestor le permite conocer qué se está haciendo, dónde, en qué cantidad, con qué calidad y a qué coste.

Los sistemas de información deben facilitar la accesibilidad a la documentación del programa de hospital a domicilio y permitir la recogida de datos y la elaboración de informes. Una particularidad de esta modalidad asistencial es que gran parte de la actividad se desarrolla fuera del hospital, y ha de conseguirse que la información pueda generarse y recuperarse en ese ámbito. De esta manera será posible generar indicadores fiables que apoyen la toma de decisiones y el control del programa. Entre los elementos que integran los sistemas de información se encuentra la historia clínica que debe cumplir con la normativa vigente y estar disponible para ser utilizada diariamente. 


\section{Objetivo}

- Disponer de sistemas de información específicos para los programas de HAD que permitan gestionar la información, emprender acciones de mejora y realizar análisis comparativos con otros programas.

\section{Conjunto mínimo básico de datos}

El Conjunto Mínimo Básico de Datos (CMBD) es un conjunto de datos administrativos y clínicos de cada paciente que ingresa en un hospital que resume la información del episodio de hospitalización cuya recogida es obligada para todos los hospitales.

Aunque existen iniciativas para aplicar sistemas de información sanitaria específicos para la atención domiciliaria basada en el hospital, todavía no se ha alcanzado consenso al respecto $(10,11)$. Los modelos existentes no recogen en su totalidad todos los aspectos relevantes de la actividad en HAD. Por ejemplo, es importante conocer no solo el domicilio habitual del paciente sino también aquel en que va a residir durante el episodio de hospitalización y la distancia a que está del hospital o del punto de atención continuada más cercano. Dentro de la denominación común de HAD se engloban modalidades asistenciales diferentes, como la atención a pacientes agudos, los cuidados paliativos, la atención a crónicos o los esquemas de hospital de día, que deberían poder individualizarse. Pese a ello, el Ministerio de Sanidad, Servicios Sociales e Igualdad ha incluido la HAD como uno de los ámbitos asistenciales para la recogida de información en el nuevo modelo de Registro de Actividad de Atención Sanitaria Especializada (12).

\section{Objetivo}

- Disponer de un registro sistemático del conjunto mínimo básico de datos (CMBD-HAD) para los pacientes de HAD que incluya elementos propios y diferenciales con respecto a la hospitalización convencional.

\section{Sistema de clasificación de los pacientes}

Los sistemas de clasificación de pacientes son modelos de cálculo que se utilizan para identificar grupos homogéneos de pacientes de perfil clínico y complejidad parecidos. La complejidad no solo hace referencia a la gravedad de la enfermedad sino también a la cantidad de recursos necesarios para atender a los pacientes.

Existen varios modelos de clasificación de los pacientes. El más conocido y utilizado es el GRD, un sistema que permite la agrupación de los pacientes en base al isoconsumo de recursos. Sin embargo, este sistema de clasificación presenta algunas dificultades para su aplicación en los programas de HAD, principalmente en los esquemas de alta precoz, ya que no siempre el motivo de ingreso en hospitalización convencional es el mismo que en HAD (13). En determinados casos, gran parte de los cuidados que precisa el paciente son proporcionados por cuidadores no sanitarios. Por ello, algunos autores han propuesto sistemas de clasificación específicos para este modelo asistencial que tengan en cuenta aspectos como la complejidad de la valoración y del tratamiento, el tiempo empleado en realizar el tratamiento y la frecuencia del mismo (15). Sin embargo, hasta la fecha ninguno de estos sistemas de clasificación ha sido utilizado de forma generalizada.

\section{Objetivo}

- Disponer de un sistema de clasificación de los pacientes para los programas de HAD, a semejanza de lo que ocurre con los pacientes convencionalmente hospitalizados, 
con el fin de medir la cantidad y calidad de la actividad, asignarles un coste y realizar benchmarking.

\section{Indicadores y cuadro de mando integral}

Los indicadores son instrumentos de medida utilizados para conocer los resultados de la actividad de una organización y el grado de consecución de los objetivos. El cuadro de mando integral está constituido por un grupo de indicadores financieros y no financieros alineados con los objetivos estratégicos de una organización.

Los indicadores permiten conocer cómo se está desarrollando el programa de HAD a distintos niveles, especialmente en lo referente a actividad, funcionamiento, consumos, costes, calidad, satisfacción y actividades en los ámbitos de la investigación y docencia. Su análisis y evaluación deben servir para emprender acciones de mejora y establecer nuevos objetivos a partir de los alcanzados con anterioridad. Del conjunto de indicadores se deben seleccionar aquellos que por su relevancia permiten un análisis dinámico y actualizado del funcionamiento y los resultados del programa y que estén en sintonía con la visión estratégica de toda lo organización. Esto permite identificar desviaciones y aplicar acciones de mejora de forma precoz.

\section{Objetivo}

- Disponer de un conjunto de los indicadores y un cuadro de mando integral común para todos los programas de HAD con el propósito de permitir comparaciones, evaluar los resultados y adoptar medidas correctoras en un proceso de mejora continua.

De forma resumida los objetivos de la línea estratégica referida a los sistemas de información del proyecto HAD 2020 son:

- Definir y consensuar los principales indicadores específicos del cuadro de mandos (CM) de la HAD.

- Diseñar y poner en producción un CM específico.

- Crear la norma de los indicadores establecidos.

- Hacer uso del CM por las unidades adheridas a SEHAD. Promover el Benchmark.

- Desarrollar un modelo de clasificación de pacientes HAD.

- Generar un CMBD específico.

- Crear un banco de datos multicéntrico.

\section{Línea 3: promoción de la investigación}

La investigación en cualquier ámbito sanitario es el elemento imprescindible que genera datos para sustentar la eficacia de la práctica clínica, incrementar la seguridad de los pacientes, garantizar la gestión eficiente de los recursos y, en definitiva, mejorar la calidad (la mejora de la calidad, la gestión eficiente de los recursos, la eficacia de la práctica clínica y la seguridad de los pacientes). Por este motivo, las autoridades, gestores y profesionales sanitarios son los responsables de diseñar estrategias que permitan generar y compartir conocimientos para que se cumpla con los estándares establecidos, y de fomentar la investigación, en el caso que nos ocupa, en HAD.

La prestación del servicio de HAD debe sustentarse en resultados y prácticas basados en la evidencia, tanto en lo que se refiere a la selección de los pacientes, como a la posterior planificación de los cuidados y manejo de las enfermedades. Debido a las características del modelo, no todas 
las guías de práctica clínica existentes son aplicables directamente a la HAD, y las organizaciones y sociedades científicas deben impulsar la elaboración de consensos y directrices específicos. Este proceso de revisión permitirá además identificar las áreas en que se carece de evidencia sólida y son más necesarias nuevas líneas de investigación.

Las primeras experiencias de HAD se produjeron hace más de 50 años, a pesar de lo cual la información sobre esta modalidad asistencial es escasa en la mayoría de países. Para paliar esta carencia, el desarrollo de un programa de HAD debe impulsar la gestión del conocimiento en un doble sentido: por un lado, adquirir una formación adecuada a las necesidades y propósitos del programa; y por otro lado, difundir los resultados de experiencias e investigaciones. La difusión debe alcanzar tanto a los profesionales implicados en la prestación del servicio como a la población subsidiaria de utilizar el mismo, población que evoluciona hacia unos niveles de información y exigencia crecientes.

\section{Objetivos}

La estrategia HAD 2020 plantea la investigación y la gestión del conocimiento en HAD en los siguientes términos:

- Desarrollar de capacidades de investigación y apoyo al proceso de gestión científica, asegurando la formación adecuada y la asignación de recursos humanos y materiales de nivel adecuado.

- Generar conocimiento científico de excelencia, potenciando la actividad investigadora de grupos multicéntricos con líneas de trabajo estables.

- Trasladar a los hospitales y a la sociedad en general, los resultados innovadores de la producción científica, que deberá tener un impacto elevado y contemplar aspectos económicos y sociales, puesto que estas son características constitutivas de la HAD.

\section{Línea 4: difusión en los decisores (profesionales y políticos)}

La estrategia HAD 2020 pretende hacer difusión de cuantas acciones, investigaciones y actividades se realicen en el marco de la estrategia a través de diversas vías y medios de comunicación.

\section{Objetivos}

- Elaboración de un libro blanco de Estándares y Recomendaciones sobre la HAD.

- Definición de objetivos para la difusión HAD. Políticos, planificadores, presidentes de sociedades científicas, asociaciones de enfermos, periodistas.

- Difusión entre los profesionales de la HAD.

- Creación de un grupo de comunicación para desarrollar los objetivos anteriores, elaborar contenidos y facilitar su divulgación interna y externa.

- Promoción de una revista especializada. Fidelizar la línea editorial de alguna revista con la publicación de trabajos sobre HAD.

\section{Línea 5: profesionales sanitarios y formación en HAD}

\section{Acreditación y formación HAD}

Las unidades de HAD en España se han ido desarrollando de forma muy heterogénea en todo el Estado, dependiendo de las necesidades de cada comunidad autónoma, de cada centro hospitalario responsable de la unidad, así como de los recursos disponibles en ese momento. 
Esta situación ha provocado que tanto la cartera de servicios que ofrecen nuestras unidades de HAD así como su composición y funcionamiento sean muy dispares tanto entre las diferentes comunidades autónomas como entre los mismos hospitales de una comunidad.

Esta heterogeneidad se refleja también tanto en los marcos legales que regulan la HAD a nivel autonómico como en los que regulan la categoría profesional del personal sanitario que forma las unidades. De esta manera de los datos obtenidos de la encuesta realizada a las unidades de HAD del Estado se deduce que sólo en el 33\% de las unidades existen iniciativas de formación continuada en HAD, sólo un 27\% de las unidades tienen una ley autonómica que las regule y sólo en el 22\% de las unidades existe una categoría profesional en HAD reconocida por su comunidad autónoma.

Toda esta situación conlleva que en el proyecto HAD 2020 clave de futuro, una de las líneas estratégicas de actuación de mayor peso sea potenciar a los profesionales de la HAD y su formación.

Para ello se han definido una serie de objetivos tanto a nivel de acreditación como en formación.

\section{Objetivos en acreditación}

- Garantizar que las unidades de HAD practiquen una atención y cuidados dentro de los estándares de excelencia.

- Evitar la variabilidad entre las diferentes unidades de HAD. Hay que intentar homogeneizar las carteras de servicio de las unidades, sin dejar de tener en cuenta ni las peculiaridades ni las necesidades asistenciales de cada comunidad autónoma ni de cada centro hospitalario. Todo ello pasa por la creación de unas guías de práctica clínica comunes a todas las unidades HAD.

- Establecer un modelo de acreditación de unidades HAD basado en los estándares oficiales de calidad.

- Incluir la estrategia HAD 2020 en el Plan Nacional de Calidad.

- Crear un grupo de trabajo para la acreditación de conocimientos avanzados en HAD formado por expertos en HAD y técnicos de la Administración. Consideramos fundamental la creación de una categoría profesional en HAD tanto para personal médico como de enfermería.

\section{Objetivos en formación}

\section{a. Formación pre-grado:}

- Incluir formación específica y acreditada en HAD, así como rotaciones de los estudiantes de medicina y enfermería por las unidades. Pensamos que es básico que ya desde la formación pregrado el estudiante médico o de enfermería considere la HAD como una alternativa a la hospitalización convencional eficaz, segura y eficiente.

b. Formación post-grado:

- Establecer rotaciones obligatorias por HAD en las especialidades de Medicina Interna, Neumología y Medicina de Familia.

- Desarrollar un plan de formación específica en HAD con cursos acreditados y con valor curricular para personal médico y de enfermería, y que permitan el desarrollo de competencias y habilidades en esta modalidad asistencial.

- Máster en HAD. 


\section{Línea 6: Financiación HAD}

La mejora de la financiación HAD e incluso en algunos casos su reconocimiento es un elemento clave para la consolidación en España. Algunos datos publicados en distintas referencias o comunicados por el propio grupo de expertos avalan el ahorro de costes de la HAD respecto a la hospitalización convencional.

Los puntos de mejora en esta línea se concentran en conocer con la mayor exactitud los costes reales de los episodios HAD respecto a los esquemas de evitación de ingreso o de alta precoz. El reconocimiento de una estructura de recursos adecuada y suficiente, ahorrará costes. En el mismo sentido la identificación de la casuística idónea para ser atendida en régimen de HAD y las prácticas más eficientes en su atención facilitarán información valiosa a los responsables de implantar nuevas unidades HAD que garantice su sostenibilidad.

\section{Objetivos}

- El proyecto HAD 2020 establece como una de sus líneas estratégicas la potenciación de estudios multicéntricos que evidencien el ahorro de costes tradicionalmente ligado a la HAD.

- Para ello, se necesita establecer los indicadores de gestión ajustados a la HAD, así como un sistema de clasificación de pacientes que valore la complejidad de los mismos.

- La HAD debe participar de modo activo en la sostenibilidad del sistema sanitario a través de su incorporación a planes estratégicos de ámbito nacional, fundamentalmente en el campo de la cronicidad y los cuidados paliativos. La correcta estratificación de los pacientes permitirá ubicar a los pacientes con necesidad de gestión de casos, en el marco de la HAD.

- En este contexto el proyecto HAD 2020 se propone realizar las siguientes acciones en el marco de la línea estratégica del desarrollo de un modelo de financiación:

- Definir y aportar información sobre costes y actividad de diversas unidades.

- Definir Escala de Unidades relativas de coste.

- Analizar y comparar modelos de financiación.

- Promover iniciativas que permitan generar evidencia sobre la sostenibilidad de la HAD.

\section{ANEXO 1: ALGUNOS ASPECTOS SOBRE FINANCIACIÓN Y HAD}

La viabilidad de un programa de HAD depende de su financiación. A su vez, la implementación de cualquier modelo de financiación debe estar basado en el conocimiento de los costes del servicio y su potencial capacidad de ahorro con respecto a la hospitalización convencional.

Los costes de un programa de HAD se obtienen a partir de los datos de contabilidad analítica de cada centro. Este análisis que resulta complejo cuando se trata de evaluar el coste por estancia en hospitalización convencional puede realizarse con relativa facilidad para el coste por estancia o por proceso de HAD. A pesar de ello, en la encuesta realizada en 2015 a las unidades de HAD españolas, el 89\% declararon no disponer de un modelo de contabilidad analítica para su programa y el 70\% no disponían de ningún tipo de información de costes (9). 


\section{Análisis de costes en hospitalización a domicilio}

El valor en la atención sanitaria está en función de los resultados de salud obtenidos, del impacto positivo sobre el paciente y no de la actividad realizada o la estructura instalada de las organizaciones sanitarias. Otro aspecto esencial a considerar es el coste económico que supone obtener dichos resultados; la sostenibilidad del sistema. Por lo tanto, el objetivo adecuado son los resultados de salud del paciente en relación con el coste total. En resumen, el valor en la atención sanitaria se define como los resultados en relación con los costes: la eficiencia (15-17).

Diversos estudios han puesto de manifiesto que los resultados en salud de la HAD son similares e incluso superiores a los de la hospitalización convencional para un grupo seleccionado de pacientes (18), a la vez que los costes resultan significativamente menores. A continuación, en la tabla 3 (19), se detallan los costes de alguno de los principales programas de HAD de ámbito nacional. En la figura 2 (20), se especifican los costes de determinados procesos infecciosos en 3 unidades HAD con programa de TADE (Tratamiento Antimicrobiano Domiciliario Endovenoso) consolidado.

Figura 2. Coste del tratamiento antimicrobiano domiciliario endovenoso (TADE) en unidades de HAD españolas: años 2012 a 2013 (20)

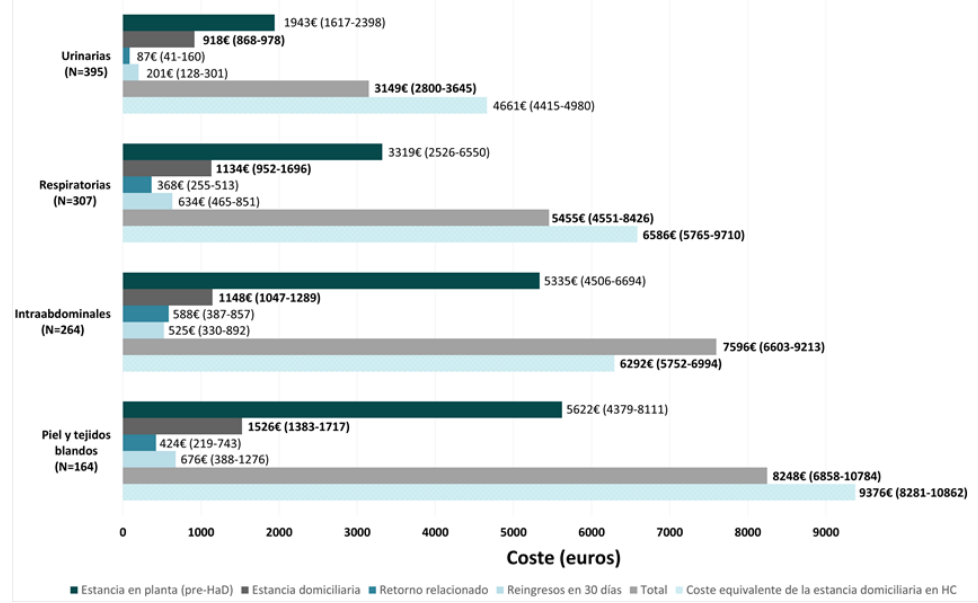

Tabla 3. Coste por estancia y por alta en unidades de Hospitalización a Domicilio (HAD) españolas: años 2006 a 2008 (19)

\begin{tabular}{|l|c|c|}
\hline CENTRO & COSTE POR ESTANCIA (€) & COSTE POR ALTA (€) \\
\hline UHD Marqués de Valdecilla & 157 & 2523 \\
\hline UHD Gregorio Marañón & 199 & 1859 \\
\hline UHD Cruces & 104 & 1529 \\
\hline
\end{tabular}




\section{Coste equivalente en hospitalización convencional: estimación de ahorro}

Desde hace años se acepta que HAD es una alternativa segura para el tratamiento de pacientes adecuadamente seleccionados. Igualmente, es ampliamente aceptado el alto nivel de satisfacción que manifiestan aquellos que han sido tratados en unidades de HAD. Esta visión positiva de la HAD frente a la hospitalización convencional, no es tan concluyente a la hora de revisar los costes comparados de la hospitalización convencional frente a la HAD.

Esta falta de concreción es debida a la ausencia de estudios de costes que evalúen de forma consistente las dos modalidades de atención, siendo necesario el establecimiento de pautas metodológicas que establezcan:

- Qué comparar: coste por estancia, coste por alta (media o GRD), coste por unidad relativa de coste.

- Con qué comparar: hospital, medicina interna, GRD.

- Qué casos incluir: todos (equivalentes a hospitalización), sin estancia previa (admission avoidance).

- Desde qué perspectiva realizar el análisis, y con qué objetivos: medir producción (actividad, complejidad), medir coste (contabilidad analítica).

El análisis comparativo de los datos de coste por estancia y coste por proceso para hospitalización convencional frente a HAD establecen datos de ahorro que alcanzan el 88\%. Sin embargo, el sesgo metodológico vuelve a posicionarse como un interrogante a considerar, por lo que esta cifra puede variar ampliamente de unos estudios a otros. Así se pone de manifiesto en la revisión de la literatura internacional y nacional donde los datos de ahorro de costes oscilan entre el 19 y $73 \%$. La estimación de ahorro, por estancia y por proceso, en unidades de HAD españolas en los años 2006 a 2008 puede consultarse en las tablas 4 y 5 (19).

Tabla 4. Estimación de ahorro por estancia en unidades de HAD españolas: años 2006 a 2008 (19)

\begin{tabular}{|l|c|c|c|}
\hline CENTRO & COSTE HAD (€) & COSTE HOSPITAL (€) & DIFERENCIA (\%) \\
\hline UHD Marqués de Valdecilla & 157 & 589 & 74 \\
\hline UHD Gregorio Marañón & 199 & 807 & 75 \\
\hline UHD Cruces & 104 & 937 & 88 \\
\hline
\end{tabular}

Tabla 5. Estimación de ahorro por proceso en unidades de HAD españolas: años 2006 a 2008 (19)

\begin{tabular}{|l|c|c|c|}
\hline CENTRO & COSTE HAD $(€)$ & COSTE HOSPITAL (€) & DIFERENCIA (\%) \\
\hline UHD Marqués de Valdecilla & 2523 & 6598 & 61 \\
\hline UHD Gregorio Marañón & 1859 & 6169 & 70 \\
\hline UHD Cruces & 1529 & 4864 & 69 \\
\hline
\end{tabular}


En un meta-análisis publicado en el año 2012 los autores observaron una fuerte evidencia de reducción de costes por episodio (diferencia media, - 1567,11\$; IC 95\%, - 2069,53 a - 1064,69; $p<0,001)$ a pesar de la gran heterogenicidad $\left(x^{2}=237.45 ; p<0.001 ; I^{2}=96 \%\right)(18)$. Se objetivaron ahorro de costes en todos los subgrupos de análisis. En general el coste medio fue del $73.5 \%$ respecto a grupo control. El análisis incluía 34 estudios de los cuales 32 concluían que el modelo de HAD era más barato.

La estimación de ahorro de los programas de HAD también se ha analizado para procedimientos específicos. Por ejemplo, el tratamiento antimicrobiano domiciliario endovenoso (TADE) puede resultar hasta un $80 \%$ menos costoso en comparación con el mismo tratamiento realizado en régimen de internamiento (21).

La eficiencia de la HAD no solo se analiza en términos monetarios. Este modelo asistencial también ha demostrado ser útil en la optimización de recursos técnicos y estructurales. En un estudio publicado en 2012 se observó una estancia media menor en los pacientes de HAD frente a la hospitalización convencional (3,3 versus 4,5 días) y un menor uso de pruebas de laboratorio y otras exploraciones diagnósticas, además de resultar un 19\% menos costoso que el modelo de ingreso tradicional (22). El número de estancias hospitalarias evitadas es otra manera de evaluar la eficiencia del modelo de HAD (21).

En los últimos años se han sucedido las publicaciones de costes en HAD y en la actualidad disponemos de un cuerpo de evidencia suficiente para poder afirmar que se trata de un modelo asistencial coste-efectivo, aunque no todos los estudios han resultado concluyentes en este sentido $(16,23-27)$.

\section{ANEXO 2: PRINCIPALES HITOS DEL PLAN ESTRATÉGICO HAD2020 EN 2016}

- Colaboración 2016 con la Agencia de Calidad y Evaluación Sanitaria de Catalunya (AQuAS) para la presentación de la experiencia HAD 2020 ante la Red de Agencias de Calidad Sanitaria de España. Marzo 2016.

- Presentación de la encuesta nacional 2016 sobre HAD. Abril 2016.

- Presentación encuesta de posicionamiento estratégico miembros SEHAD 2016. Abril 2016.

- Aprobación Plan Estratégico HAD2020. Sesión plenaria del XIII Congreso Nacional de HAD. Validación de las líneas de actuación 2017-2020. Las Palmas, 23 de abril 2016.

- Primera reunión con responsables del MSSSI. Líneas de trabajo: formación y acreditación profesionales HAD y Modelo asistencial común. Julio 2016.

- Formación de grupos de trabajo específicos. Julio-septiembre 2016.

- Acuerdo de colaboración SEHAD con la Revista HAD. Octubre 2016.

- Acuerdo colaboración con SEPAR. Octubre 2016.

- Acuerdo colaboración con SEIMC. Noviembre 2016.

- Acuerdo colaboración con SECPAL. Diciembre 2016.

- Acuerdo colaboración SENPE. Diciembre 2016.

- Premio Mejores Ideas 2016 al Plan Estratégico HAD 2020: clave de futuro. Diario Médico. Noviembre 2016

- Primera versión del Glosario terminológico HAD. Diciembre 2016 


\section{BIBLIOGRAFÍA}

1. Cherkasky M. The Montefiore Hospital Home Care Program. Am J Public Health Nations Health. 1949;39(2):163-6. PMID: 18111592

2. Organización Mundial de la Salud (OMS). Función de los Hospitales en la asistencia médica ambulatoria y domiciliaria. Ginebra, Suiza: 1959. Informes Técnicos n 176.

3. Orden Ministerial, de 1 de marzo de 1985, por la que se aprueba el Reglamento General de Estructura, Organización y Funcionamiento de los Hospitales de la Seguridad Social. Boletín Oficial del Estado, n 5, (5 de marzo de 1985). Artículo 9.

4. Real Decreto 521/1987, de 15 de abril, por el que se aprueba el Reglamento sobre Estructura, Organización y Funcionamiento de los Hospitales gestionados por el Instituto Nacional de la Salud. Boletín Oficial del Estado, no 91, (16 de abril de 1987).

5. Ley 14/1986, de 25 de abril, General de Sanidad. Boletín Oficial del Estado, nº 102, (29 de abril de 1986). Artículo 18.

6. Real Decreto 1030/2006, de 15 de septiembre, por el que se establece la cartera de servicios comunes del Sistema Nacional de Salud y el procedimiento para su actualización. Boletín Oficial del Estado, $n^{\circ}$ 222, (16 de septiembre de 2006). Anexo III, punto 4: Hospitalización a Domicilio.

7. Real Decreto 69/2015, de 6 de febrero, por el que se regula el Registro de Actividad de Atención Sanitaria Especializada. Boletín Oficial del Estado, nº 35, (10 de febrero de 2015).

8. Antón Botella F. Análisis estratégico de las unidades de HAD en España en 2015. En: Ponencia al $13^{\circ}$ Congreso Nacional de la SEHAD, 21 al 23 de abril de 2016. Las Palmas de Gran Canaria: Sociedad Española de Hospitalización a Domicilio; 2016.

9. Massa B. Clave de futuro: hoja de ruta hacia las HAD del 2020. En: Ponencia al $13^{\circ}$ Congreso Nacional de la Sociedad Española de Hospitalización a Domicilio (SEHAD), 21 al 23 de abril. Las Palmas de Gran Canaria: SEHAD; 2016.

10. Morris JN, Fries BE, Steel K, Ikegami N, Bernabei R, Carpenter Gl, et al. Comprehensive clinical assessment in community setting: applicability of the MDS-HC. J Am Geriatr Soc. 1997;45(8):1017-24. PMID: 9256857

11. Stoker J. OASIS as a mandatory Medicare requirement. Home Healthc Nurse. 1998;16(9):595. PMID: 9807312

12. Real Decreto 69/2015, de 6 de febrero, por el que se regula el Registro de Actividad de Atención Sanitaria Especializada. Boletín Oficial del Estado, n 35, (10 de febrero de 2015). Sección I, página 10789.

13. Duckett SJ, Jackson T. Casemix classification for outpatient services based on episodes of care. Med J Aust. 1993;158(7):489-92. PMID: 8379985

14. Santamaria N, Daly S, Addicott R, Clayton L. The development, validity and reliability of the Hospital in the Home Dependency Scale. Aust J Adv Nurs. 2001;18(4):8-14. PMID: 11878551

15. González Ramallo V, Valdivieso B, Ruiz V. Hospitalización a Domicilio. Med Clin (Barc) 2002; 118: 659-64. PMID: 12028904

16. Pericás JM, Aibar J, Soler N, López-Soto A, Sanclemente-Ansó C, Bosch X. Should alternatives to conventional hospitalisation be promoted in an era of financial constraint? Eur J Clin Invest. 2013;43(6):602-15. DOI: 10.1111/eci.12087; PMID: 23590593 
17. Porter ME. What is value in health care? N Engl J Med. 2010;363(26):2477-81. DOI: 10.1056/ NEJMp1011024; PMID: 21142528

18. Caplan GA, Sulaiman NS, Mangin DA, Aimonino Ricauda N, Wilson AD, Barclay L. A metaanalysis of "hospital in the home". Med J Aust. 2012;197(9):512-9. DOI: 10.5694/mja12.10480; PMID: 23121588

19. González Ramallo V. Estudios de costes de la actividad realizada en las unidades de hospitalización a domicilio de España. En: XXXIV Congreso Nacional de la Sociedad Española de Medicina Interna (SEMI), 21 al 23 de noviembre. Málaga: SEMI; 2013.

20. Mirón M, Estrada O, González-Ramallo VJ, Mujal A, Forné C, Uría E, et al. Costes del tratamiento antimicrobiano domiciliario endovenoso (TADE) en España por tipo de infección. En: XX Congreso Nacional de la Sociedad Española de Enfermedades Infecciosas y Microbiología Clínica (SEIMC), 26 al 28 de mayo. Barcelona: SEIMC; 2016.

21. Mujal A, Estrada O, Mirón-Rubio M, González-Ramallo VJ, Forné C, Uría E, et al. Costs of outpatient parenteral antibiotic therapy (OPAT) in Spain: A multicenter economic evaluation (the Spanish OPAT registry). In: 26th European Congress of Clinical Microbiology and Infectious Diseases, 9 a 12 abril. Amsterdam: European Society of Clinical Microbiology and Infectious Diseases; 2016.

22. Cryer L, Shannon SB, Van Amsterdam M, Leff B. Costs for 'hospital at home' patients were 19 percent lower, with equal or better outcomes compared to similar inpatients. Health Aff (MiIIwood). 2012;31(6):1237-43. DOI: 10.1377/hlthaff.2011.1132; PMID: 22665835

23. Hernandez C, Casas A, Escarrabill J, Alonso J, Puig-Junoy J, Farrero E, et al. Home hospitalisation of exacerbated chronic obstructive pulmonary disease patients. Eur Respir J. 2003;21(1):5867. DOI: 10.1183/09031936.03.00015603; PMID: 12570110

24. Shepperd S, Doll H, Angus RM, Clarke MJ, lliffe S, Kalra L, et al. Avoiding hospital admission through provision of hospital care at home: a systematic review and meta-analysis of individual patient data. CMAJ. 2009;180(2):175-82. DOI: 10.1503/cmaj.081491; PMID: 19153394

25. Mendoza H, Martín MJ, García A, Arós F, Aizpuru F, Regalado De Los Cobos J, et al. 'Hospital at home' care model as an effective alternative in the management of decompensated chronic heart failure. Eur J Heart Fail. 2009;11(12):1208-13. DOI: 10.1093/eurjhf/hfp143; PMID: 19875400

26. Jiménez S, Aguiló S, Antolín A, Coll-Vinent B, Miró O, Sánchez M. Hospitalización a domicilio directamente desde urgencias: una alternativa eficiente a la hospitalización convencional. Med Clin (Barc). 2011;137(13):587-90. DOI: 10.1016/j.medcli.2011.06.012; PMID: 21940003

27. Varney J, Weiland TJ, Jelinek G. Efficacy of hospital in the home services providing care for patients admitted from emergency departments: an integrative review. Int J Evid Based Healthc. 2014;12(2):128-41. DOI: 10.1097/XEB.0000000000000011; PMID: 24945961 